\title{
Synthesis of a small library containing substituted pyrazoles
}

\author{
Xue L. Ren ${ }^{a}$, Hua B. Li ${ }^{b}$, Chao Wu ${ }^{b}$, and Hua Z. Yang*b \\ ${ }^{a}$ School of Engineering and Technology, Tianjin University, Tianjin 300072, China \\ ${ }^{b}$ Institute of Elemento-Organic Chemistry, Nankai University, Tianjin 300071, China \\ E-mail: drs1998@126.com
}

(received 01 Jun 05; accepted 01 Sep 05; published on the web 05 Sep 05)

\begin{abstract}
Prompted by the observation that many pyrazole derivatives are used as herbicides, a small combinatorial library containing pyrazolylpyrazoles and pyrazolo[1,5-a]pyrimidines was synthesized by traditional organic synthesis and parallel-liquid-phase combinatorial synthesis. The structures of compounds were confirmed by ${ }^{1} \mathrm{H}$ NMR, LC and elemental analysis.
\end{abstract}

Keywords: Pyrazolylpyrazoles, pyrazolo[1,5-a]pyrimidines, parallel-liquid-phase synthesis, combinatorial library, synthesis

\section{Introduction}

Combinatorial chemistry is widely used in pharmaceutical research as a powerful tool for fast identification of novel therapeutic agents in drug discovery. Liquid phase synthesis is a methodology where all reactions are carried out in their own separate reaction vessels. It is a typical parallel synthesis and it is suitable for almost all kinds of reactions in a relatively large scale. In this field, multicomponent condensation reactions have been utilized very efficiently in conjugation with combinatorial chemistry to prepare large collections of molecules in a short reaction sequence.

Heterocyclic compounds represent an important class of biologically active molecules. Specifically, those containing the pyrazole nucleus have been shown to possess high biological activities as herbicides, fungicides, analgesics, etc. Herbicidally active pyrazolylpyrazoles have been reported previously. ${ }^{1}$ Pyrazolo[1,5-a]pyrimidines are good inhibitors of KDR kinase. ${ }^{2}$

This article describes a small combinatorial library containing pyrazolylpyrazoles and pyrazolo[1,5-a]pyrimidines synthesized by traditional organic synthesis and by parallel-liquidphase combinatorial synthesis (Scheme 1). 


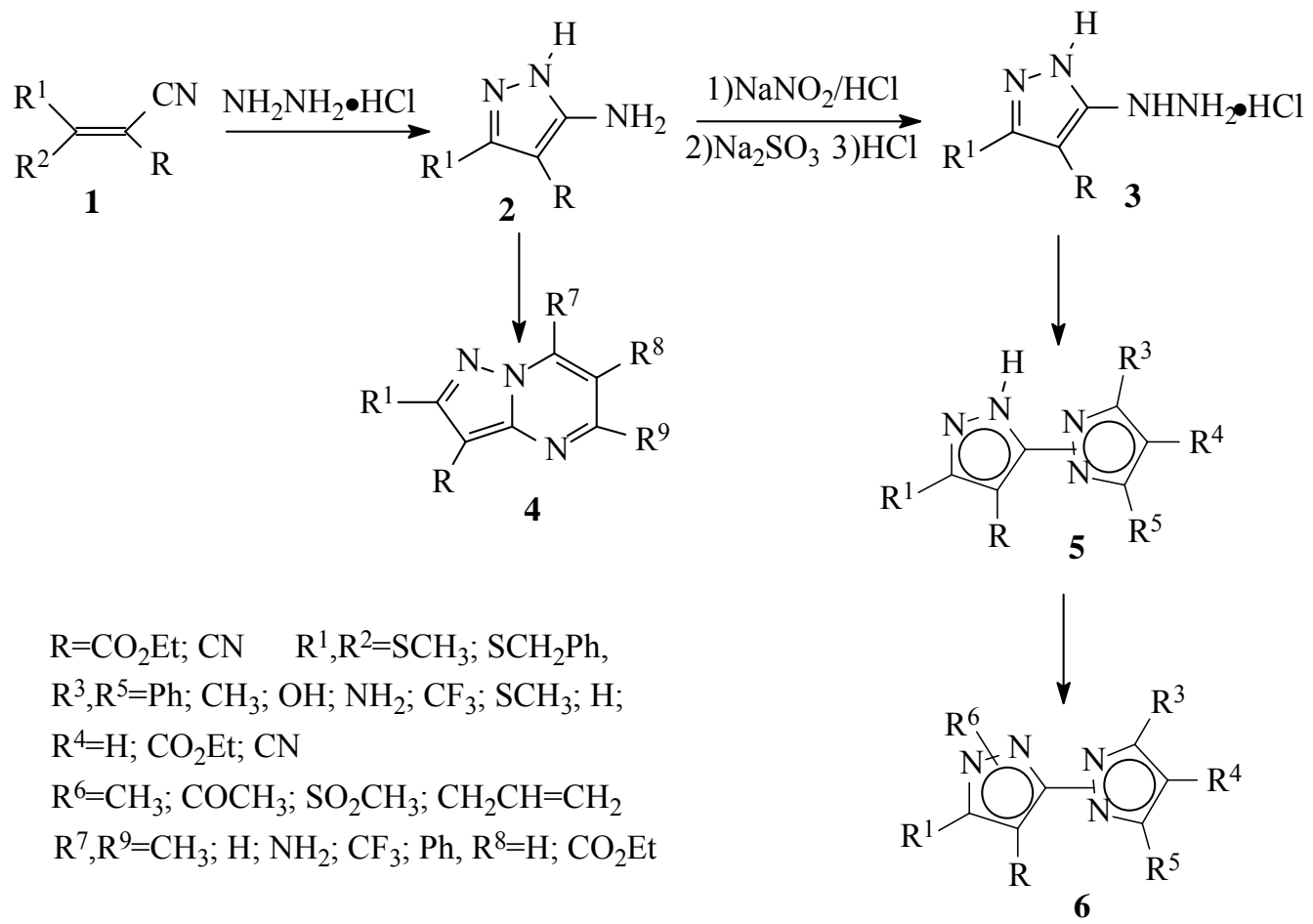

\section{Scheme 1}

\section{Results and Discussion}

A building block for many of the compounds was 5-amino- $1 H$-pyrazole (2), which was obtained by treating substituted acrylonitrile (1) with one equivalent of hydrazine hydrate in ethanol and tetrahydrofuran at room temperature. ${ }^{3}$ With increasing the amount of hydrazine hydrate used, a few byproducts, such as carbohydrazides, occurred in the reaction. Therefore, increasing the amount of hydrazine hydrate was harmful to the reaction.

Cyclization of $\mathbf{2}$ with bifunction compounds in glacial acetic acid gave the desired pyrazolo[1,5-a]pyrimidines of general formula 4, which might result in the production of two positional isomers (Scheme 2).,

The diazonium salt of $\mathbf{2}$ was achieved by the diazotization of the appropriate pyrazolylamine 2 in a solution of hydrochloric acid with a saturated solution of sodium nitrite in water for $1 \mathrm{~h}$. Then the diazonium salt was reduced with sodium sulfite to achieve corresponding pyrazole hydrazine salt 3 which was used in the next reaction without further purification. ${ }^{5}$

From the key intermediate 3 the $1 H$-pyrazolylpyrazoles of general formula 5 were prepared by cyclocondensation with one equivalent of the appropriate bifunctional compounds like diketos, substituted acrylonitrile, etc. ${ }^{6-8}$ The synthetic pathway allowed the formation of a mixture of two isomers that were not separated (Scheme 3). 
1-Substituted-pyrazolylpyrazoles of general formula 6 were obtained by a further substitution of compounds 5. N-substitution in the pyrazole ring showed different results depending on the groups in other positions, so there might be $2 \sim 4$ isomers in one reaction (Scheme 4). ${ }^{9,10}$

To rapidly generate substituted pyrazoles with multiplicity of structures and discover the structure-activity relationships of pyrazole derivates, we chose to consider position isomers as groups. In this work, 30 daughter-libraries containing 70 compounds in total were obtained.

The existence of isomers was reflected in the ${ }^{1} \mathrm{H}$ NMR spectra of the products. The isomers of compounds showed similar analytical and ${ }^{1} \mathrm{H}$ NMR spectroscopic data where many of the hydrogen signals appeared in pairs, which confirmed the structures of the isomeric pyrazole derivatives. The existence of isomers was also confirmed by GCD (Gas Chromatography Directory) and liquid chromatogram.

Yields, number of isomers, ${ }^{1} \mathrm{H}$ NMR spectral data and elemental analyses of compounds 4, 5 and 6 are given in Table 1, 2 and 3 respectively. Analyses indicated by the symbols of the elements or functions were within $\pm 0.4{ }^{\circ} \mathrm{C}$ of theoretical values.<smiles>[R]c1nc2c([R])c([R])nn2c([R])c1[R]</smiles>

4-1<smiles>[R]c1nc2c([R])c([R])nn2c([R])c1[R7]</smiles>

4-2

\section{Scheme 2}<smiles>[R]c1n[nH]c(-n2nc([R])c([R])c2[R])c1[R]</smiles>

5-1<smiles>[R]c1n[nH]c(-n2nc([R])c([R])c2[R])c1[R]</smiles>

5-2

\section{Scheme 3}<smiles>[R]c1nn([R7])c(-n2nc([R])c([R])c2[R])c1[R]</smiles>

6-1<smiles>[R]c1nn([R7])c(-n2nc([R])c([R])c2[R])c1[R]</smiles>

6-2<smiles>[R]c1nn(-c2nn([R8])c([R])c2[R])c([R8])c1[R]</smiles>

6-3<smiles></smiles>

6-4

\section{Scheme 4}


Table 1. Yield, spectroscopic data and elemental analyses of 4

\begin{tabular}{|c|c|c|c|c|c|c|c|}
\hline \multirow[t]{2}{*}{ No } & \multirow[t]{2}{*}{ Structure } & \multirow{2}{*}{$\begin{array}{l}\text { Yield } \\
(\%)\end{array}$} & \multirow{2}{*}{$\begin{array}{c}\mathrm{Nu} \\
\mathrm{m}\end{array}$} & \multirow[t]{2}{*}{${ }^{1} \mathrm{HNMR}(\delta \mathrm{ppm} \mathrm{CDCl} 3)$} & \multicolumn{3}{|c|}{$\begin{array}{l}\text { Elemental analysis } \\
\text { (Found/Calc.\%) }\end{array}$} \\
\hline & & & & & $\mathrm{C}$ & $\mathrm{H}$ & $\mathrm{N}$ \\
\hline \multirow{2}{*}{ 4a } & \multirow{2}{*}{$\begin{array}{c}\mathrm{R}=\mathrm{CO}_{2} \mathrm{Et} ; \mathrm{R}^{1}=\mathrm{CH}_{3} \mathrm{~S} ; \\
\mathrm{R}^{7}, \mathrm{R}^{9}=\mathrm{CH}_{3} ; \mathrm{R}^{8}=\mathrm{H}\end{array}$} & \multirow{2}{*}{97.1} & \multirow{2}{*}{1} & $\begin{array}{l}1.382-1.453(3 \mathrm{H}) ; 2.599(\mathrm{~s} \\
3 \mathrm{H}) ; 2.671(\mathrm{~s}, 3 \mathrm{H}) ; 2.691(\mathrm{~s}\end{array}$ & 54.77 & 5.04 & 15.85 \\
\hline & & & & $\begin{array}{l}3 \mathrm{H}) ; 4.411-4.447(\mathrm{q}, 2 \mathrm{H}) ; 6.645 \\
(\mathrm{~s}, 1 \mathrm{H})\end{array}$ & 54.74 & 4.98 & 15.96 \\
\hline \multirow[b]{2}{*}{$4 b$} & \multirow{2}{*}{$\begin{array}{c}\mathrm{R}=\mathrm{CN} ; \mathrm{R}^{1}=\mathrm{PhCH}_{2} \mathrm{~S} \\
\mathrm{R}^{7}, \mathrm{R}^{9}=\mathrm{CH}_{3} ; \mathrm{R}^{8}=\mathrm{H}\end{array}$} & \multirow[b]{2}{*}{96.4} & \multirow[b]{2}{*}{1} & $2.592(\mathrm{~s}, 3 \mathrm{H}) ; 2.715(\mathrm{~s}, 3 \mathrm{H})$ & 64.99 & 5.05 & 19.17 \\
\hline & & & & $\begin{array}{l}4.453(\mathrm{~s}, 2 \mathrm{H}) ; 6.680(\mathrm{~s}, 1 \mathrm{H}) \\
7.240-7.403(\mathrm{~m}, 5 \mathrm{H})\end{array}$ & 65.28 & 4.79 & 19.03 \\
\hline \multirow{2}{*}{$4 c$} & $\mathrm{R}=\mathrm{CO}_{2} \mathrm{Et} ; \mathrm{R}^{1}=\mathrm{CH}_{3} \mathrm{~S}$ & \multirow{2}{*}{92.4} & \multirow{2}{*}{2} & $1.131-1.201(3 \mathrm{H}) ; 1.346-1.416$ & 47.47 & 4.12 & 25.39 \\
\hline & $\mathrm{R}^{7}, \mathrm{R}^{9}=\mathrm{H}, \mathrm{NH}_{2} ; \mathrm{R}^{8}=\mathrm{CO}_{2} \mathrm{Et}$ & & & $\begin{array}{l}(3 \mathrm{H}) ; 2.582(\mathrm{~s}, 3 \mathrm{H}) ; 4.321(\mathrm{~m} \\
4 \mathrm{H}) ; 8.150(\mathrm{~s}, 1 \mathrm{H})\end{array}$ & 47.65 & 4.00 & 25.26 \\
\hline \multirow{2}{*}{ 4d } & $\mathrm{R}=\mathrm{CN} ; \mathrm{R}^{1}=\mathrm{CH}_{3} \mathrm{~S}$ & \multirow{2}{*}{96.4} & \multirow{2}{*}{1} & $2.579-2.691(\mathrm{~m}, 9 \mathrm{H}) ; 6.656-$ & 55.16 & 4.44 & 25.68 \\
\hline & $\mathrm{R}^{7}, \mathrm{R}^{9}=\mathrm{CH}_{3} ; \mathrm{R}^{8}=\mathrm{H}$ & & & $7.224(\mathrm{~d}, 1 \mathrm{H})$ & 55.03 & 4.62 & 25.67 \\
\hline \multirow[b]{2}{*}{$4 e$} & $\mathrm{R}=\mathrm{CN} ; \mathrm{R}^{1}=\mathrm{CH}_{3} \mathrm{~S}$ & \multirow[b]{2}{*}{25.0} & \multirow[b]{2}{*}{2} & 1.344-1.414 (m, 3H); 2.673- & 41.80 & 3.05 & 20.32 \\
\hline & $\begin{array}{c}\mathrm{R}^{7}, \mathrm{R}^{9}=\mathrm{NH}_{3}, \mathrm{CF}_{3} ; \mathrm{R}^{8}=\mathrm{CO}_{2} \mathrm{E} \\
\mathrm{t}\end{array}$ & & & $\begin{array}{l}2.845(\mathrm{~d}, 3 \mathrm{H}) ; 4.378-4.417(\mathrm{~m}, \\
2 \mathrm{H})\end{array}$ & 41.74 & 2.92 & 20.28 \\
\hline \multirow{2}{*}{$4 \mathrm{f}$} & $\mathrm{R}=\mathrm{CN} ; \mathrm{R}^{1}=\mathrm{CH}_{3} \mathrm{~S}$ & \multirow{2}{*}{89.3} & & $2.610-2.665(\mathrm{~d}, 3 \mathrm{H}) ; 2.696-$ & 64.21 & 4.35 & 19.93 \\
\hline & $\mathrm{R}^{7}, \mathrm{R}^{9}=\mathrm{CH}_{3}, \mathrm{Ph} ; \mathrm{R}^{8}=\mathrm{H}$ & & & $\begin{array}{l}2.804(\mathrm{~d}, 3 \mathrm{H}) ; 6.878-7.224(\mathrm{~d} \\
1 \mathrm{H}) ; 7.500-8.129(\mathrm{~m}, 5 \mathrm{H})\end{array}$ & 64.26 & 4.31 & 19.98 \\
\hline \multirow{2}{*}{$4 \mathrm{~g}$} & $\mathrm{R}=\mathrm{CN} ; \mathrm{R}^{1}=\mathrm{CH}_{3} \mathrm{~S}$ & \multirow{2}{*}{18.2} & \multirow[b]{2}{*}{2} & $1.359-1.429(\mathrm{t}, 3 \mathrm{H}) ; 2.682(\mathrm{~s}$ & 47.69 & 3.92 & 25.32 \\
\hline & $\mathrm{R}^{7}, \mathrm{R}^{9}=\mathrm{NH}_{2}, \mathrm{H} ; \mathrm{R}^{8}=\mathrm{CO}_{2} \mathrm{Et}$ & & & $\begin{array}{l}3 \mathrm{H}) ; 4.370-4.408(\mathrm{q}, 4 \mathrm{H}) ; 8.190 \\
(\mathrm{~s}, 1 \mathrm{H})\end{array}$ & 47.64 & 4.00 & 25.25 \\
\hline
\end{tabular}


Table 2. Yield, spectroscopic data and elemental analyses of 5

\begin{tabular}{|c|c|c|c|c|c|c|c|}
\hline \multirow[t]{2}{*}{ No } & \multirow[t]{2}{*}{ Structure } & \multirow{2}{*}{$\begin{array}{l}\text { Yield } \\
(\%)\end{array}$} & \multirow{2}{*}{$\begin{array}{c}\mathrm{Nu} \\
\mathrm{m}\end{array}$} & \multirow[t]{2}{*}{$\left.{ }^{1} \mathrm{HNMR}(\delta \mathrm{ppm} \mathrm{CDCl})_{3}\right)$} & \multicolumn{3}{|c|}{$\begin{array}{l}\text { Elemental analysis } \\
\text { (Found/Calc.\%) }\end{array}$} \\
\hline & & & & & $\mathrm{C}$ & $\mathrm{H}$ & $\mathrm{N}$ \\
\hline $5 a$ & $\begin{array}{l}\mathrm{R}=\mathrm{CO}_{2} \mathrm{Et} ; \mathrm{R}^{1}=\mathrm{SMe} \\
\mathrm{R}^{3}, \mathrm{R}^{5}=\mathrm{Ph}, \mathrm{Me} ; \mathrm{R}^{4}=\mathrm{H}\end{array}$ & 71.5 & 2 & $\begin{array}{l}1.027-1.074(\mathrm{t}, 3 \mathrm{H}) ; 2.357(\mathrm{~s}, 3 \mathrm{H},) ; \\
2.491(\mathrm{~s}, 3 \mathrm{H}) ; 3.937-4.008(\mathrm{q}, 2 \mathrm{H}) \\
6.338(\mathrm{~s}, 1 \mathrm{H})\end{array}$ & 59.54 & 5.28 & 16.24 \\
\hline $5 b$ & $\begin{array}{c}\mathrm{R}=\mathrm{CO}_{2} \mathrm{Et} ; \mathrm{R}^{1}=\mathrm{SMe} \\
\mathrm{R}^{3}, \mathrm{R}^{5}=\mathrm{Me} ; \mathrm{R}^{4}=\mathrm{H}\end{array}$ & 73.7 & 1 & $\begin{array}{l}1.118-1.189(\mathrm{t}, 3 \mathrm{H}) ; 2.176(\mathrm{~s}, 3 \mathrm{H},) ; \\
2.446(\mathrm{~s}, 3 \mathrm{H}) ; 2.679(\mathrm{~s}, 3 \mathrm{H}) ; 4.130- \\
4.164(\mathrm{q}, 2 \mathrm{H}) ; 6.143(\mathrm{~s}, 1 \mathrm{H})\end{array}$ & 51.45 & 5.74 & 19.98 \\
\hline $5 c$ & $\begin{array}{c}\mathrm{R}=\mathrm{CO}_{2} \mathrm{Et} ; \mathrm{R}^{1}=\mathrm{SMe} \\
\mathrm{R}^{3}, \mathrm{R}^{5}=\mathrm{OH}, \mathrm{Me} \\
\mathrm{R}^{4}=\mathrm{H}\end{array}$ & 68.4 & 2 & $\begin{array}{l}1.313-1.349(\mathrm{~m}, 3 \mathrm{H}) ; 2.544-2.568(\mathrm{~d}, \\
3 \mathrm{H}) ; 3.889-3.903(\mathrm{~d}, 3 \mathrm{H}) ; 4.248(\mathrm{~m} \\
2 \mathrm{H}) ; 7.820(\mathrm{~d}, 1 \mathrm{H})\end{array}$ & 46.80 & 5.14 & 19.85 \\
\hline $5 d$ & $\begin{array}{c}\mathrm{R}=\mathrm{CO}_{2} \mathrm{Et} ; \mathrm{R}^{1}=\mathrm{SMe} \\
\mathrm{R}^{3}, \mathrm{R}^{5}=\mathrm{NH}_{2}, \mathrm{H} \\
\mathrm{R}^{4}=\mathrm{CO}_{2} \mathrm{Et}\end{array}$ & 74.6 & 2 & $\begin{array}{l}1.302-1.389(\mathrm{~m}, 6 \mathrm{H}) ; 2.779(\mathrm{~s}, 3 \mathrm{H}) \\
4.287-4.357(\mathrm{~m}, 4 \mathrm{H}) ; 8.054(\mathrm{~s}, 1 \mathrm{H})\end{array}$ & 45.96 & 4.96 & 20.67 \\
\hline $5 e$ & $\begin{array}{c}\mathrm{R}=\mathrm{CN} ; \mathrm{R}^{1}=\mathrm{SCH}_{2} \mathrm{Ph} \\
\quad \mathrm{R}^{3}, \mathrm{R}^{5}=\mathrm{Me} ; \mathrm{R}^{4}=\mathrm{H}\end{array}$ & 71.5 & 1 & $\begin{array}{l}2.657(\mathrm{~s}, 3 \mathrm{H}) ; 2.778(\mathrm{~s}, 3 \mathrm{H}) ; 4.690(\mathrm{~s} \\
2 \mathrm{H}) ; 6.830(\mathrm{~s}, 1 \mathrm{H}) ; 7.196-7.373(\mathrm{~m} \\
5 \mathrm{H})\end{array}$ & 62.18 & 4.85 & 22.79 \\
\hline $5 f$ & $\begin{array}{c}\mathrm{R}=\mathrm{CO}_{2} \mathrm{Et} ; \mathrm{R}^{1}=\mathrm{SMe} \\
\mathrm{R}^{3}, \mathrm{R}^{5}=\mathrm{NH}_{2}, \mathrm{CF}_{3} \\
\mathrm{R}^{4}=\mathrm{CO}_{2} \mathrm{Et}\end{array}$ & 82.3 & 2 & $\begin{array}{l}1.150-1.197(\mathrm{t}, 3 \mathrm{H}) ; 1.326-1.373(\mathrm{t}, \\
3 \mathrm{H}) ; 2.503(\mathrm{~s}, 3 \mathrm{H}) ; 4.149-4.220(\mathrm{q}, \\
2 \mathrm{H}) ; 4.267-4.339(\mathrm{q}, 2 \mathrm{H}) ; 5.820(2 \mathrm{H})\end{array}$ & 41.28 & 3.96 & 17.19 \\
\hline $5 g$ & $\begin{aligned} \mathrm{R} & =\mathrm{CO}_{2} \mathrm{Et} ; \\
\mathrm{R}^{1} & =\mathrm{SCH}_{2} \mathrm{Ph} ; \\
\mathrm{R}^{3}, \mathrm{R}^{5} & =\mathrm{CH}_{3} ; \mathrm{R}^{4}=\mathrm{H}\end{aligned}$ & 54.2 & 1 & $\begin{array}{l}1.083-1.152(\mathrm{t}, 3 \mathrm{H}) ; 2.119(\mathrm{~s}, 3 \mathrm{H}) ; \\
2.309(\mathrm{~s}, 3 \mathrm{H}) ; 4.097-4.133(\mathrm{q}, 2 \mathrm{H}) ; \\
4.331(\mathrm{~s}, 2 \mathrm{H}) ; 6.009(\mathrm{~s}, 1 \mathrm{H}) ; 5.820 \\
(2 \mathrm{H})\end{array}$ & 60.77 & 5.02 & 15.64 \\
\hline $5 h$ & $\begin{array}{c}\mathrm{R}=\mathrm{CO}_{2} \mathrm{Et} ; \mathrm{R}^{1}=\mathrm{SMe} \\
\mathrm{R}^{3}, \mathrm{R}^{5}=\mathrm{SMe}, \mathrm{NH}_{2} \\
\mathrm{R}^{4}=\mathrm{CN}\end{array}$ & 77.3 & 2 & $\begin{array}{l}1.317-1.365(\mathrm{t}, 3 \mathrm{H}) ; 2.596-2.602(\mathrm{~d}, \\
3 \mathrm{H}) ; 2.746(\mathrm{~s}, 3 \mathrm{H}) ; 4.243-4.314(\mathrm{q} \\
2 \mathrm{H}) ; 7.259(1 \mathrm{H})\end{array}$ & 42.49 & 4.19 & 24.52 \\
\hline $5 \mathbf{i}$ & $\begin{array}{l}\mathrm{R}=\mathrm{CO}_{2} \mathrm{Et} ; \mathrm{R}^{1}=\mathrm{SMe} \\
\mathrm{R}^{3}, \mathrm{R}^{5}=\mathrm{H}, \mathrm{CF}_{3} ; \mathrm{R}^{4}=\mathrm{H}\end{array}$ & 89.7 & 2 & $\begin{array}{l}1.358-1.420(\mathrm{t}, 3 \mathrm{H}) ; 2.538(\mathrm{~s}, 3 \mathrm{H}) \\
3.323-3.386(\mathrm{~d}, 1 \mathrm{H}) ; 3.560-3.626(\mathrm{~d}, \\
1 \mathrm{H}) ; 4.313-4.384(\mathrm{q}, 2 \mathrm{H})\end{array}$ & 41.25 & 3.46 & 17.49 \\
\hline $5 \mathbf{j}$ & $\begin{array}{c}\mathrm{R}=\mathrm{CO}_{2} \mathrm{Et} \\
\mathrm{R}^{1}=\mathrm{SCH}_{2} \mathrm{Ph} \\
\mathrm{R}^{3}, \mathrm{R}^{5}=\mathrm{NH}_{2}, \mathrm{H} \\
\mathrm{R}^{4}=\mathrm{CO}_{2} \mathrm{Et}\end{array}$ & 68.1 & 2 & $\begin{array}{l}1.328-1.389(\mathrm{~m}, 3 \mathrm{H}) ; 2.204(\mathrm{~s}, 2 \mathrm{H}) ; \\
4.162-4.339(\mathrm{q}, 2 \mathrm{H}) ; 7.210-7.324(\mathrm{~m}, \\
5 \mathrm{H}) ; 7.815(\mathrm{~s}, 1 \mathrm{H})\end{array}$ & 55.42 & 4.38 & 22.81 \\
\hline
\end{tabular}


Table 3. Yield, spectroscopic data and elemental analyses of 6

\begin{tabular}{|c|c|c|c|c|c|c|c|}
\hline \multirow[t]{2}{*}{ No } & \multirow[t]{2}{*}{ Structure } & \multirow{2}{*}{$\begin{array}{c}\text { Yield } \\
(\%)\end{array}$} & \multirow{2}{*}{ Num } & \multirow[t]{2}{*}{${ }^{1} \mathrm{HNMR}\left(\delta \mathrm{ppm} \mathrm{CDCl}_{3}\right)$} & \multicolumn{3}{|c|}{$\begin{array}{c}\text { Elemental analysis } \\
\text { (Found/Calc.\%) }\end{array}$} \\
\hline & & & & & $\mathrm{C}$ & $\mathrm{H}$ & $\mathrm{N}$ \\
\hline $6 a$ & $\begin{array}{c}\mathrm{R}=\mathrm{CO}_{2} \mathrm{Et} ; \mathrm{R}^{1}=\mathrm{SMe} \\
\mathrm{R}^{3}, \mathrm{R}^{5}=\mathrm{Ph}, \mathrm{CH}_{3} \\
\mathrm{R}^{4}=\mathrm{H} ; \mathrm{R}^{6}=\mathrm{CH}_{3}\end{array}$ & 98.2 & 4 & $\begin{array}{l}1.039-1.086(\mathrm{~m}, 3 \mathrm{H}) ; 2.377(\mathrm{~s} \\
3 \mathrm{H}) ; 2.522(\mathrm{~s}, 3 \mathrm{H}) ; 3.593(\mathrm{~s} \\
3 \mathrm{H}) ; 4.004-4.050(\mathrm{~m}, 2 \mathrm{H}) ; 6.401 \\
(\mathrm{~s}, 1 \mathrm{H}) ; 7.132-7.307(\mathrm{~m}, 5 \mathrm{H})\end{array}$ & 60.58 & 5.49 & 16.00 \\
\hline $6 b$ & $\begin{array}{c}\mathrm{R}=\mathrm{CO}_{2} \mathrm{Et} ; \mathrm{R}^{1}=\mathrm{SMe} \\
\mathrm{R}^{3}, \mathrm{R}^{5}=\mathrm{Ph}, \mathrm{CH}_{3} \\
\mathrm{R}^{4}=\mathrm{H} ; \mathrm{R}^{6}=\mathrm{COCH}_{3}\end{array}$ & 91.6 & 4 & $\begin{array}{l}1.033-1.130(\mathrm{~m}, 3 \mathrm{H}) ; 2.361- \\
2.371(\mathrm{~d}, 3 \mathrm{H}) ; 2.525-2.539(\mathrm{~d} \\
3 \mathrm{H}) ; 3.583(\mathrm{~s}, 3 \mathrm{H}) ; 3.971-4.134 \\
(\mathrm{~m}, 2 \mathrm{H}) ; 6.351(\mathrm{~s}, 1 \mathrm{H}) ; 7.189- \\
7.299(\mathrm{~m}, 5 \mathrm{H})\end{array}$ & 59.36 & 5.24 & 14.57 \\
\hline $6 c$ & $\begin{array}{c}\mathrm{R}=\mathrm{CO}_{2} \mathrm{Et} ; \mathrm{R}^{1}=\mathrm{SMe} \\
\mathrm{R}^{3}, \mathrm{R}^{5}=\mathrm{CH}_{3} \\
\mathrm{R}^{4}=\mathrm{H} ; \mathrm{R}^{6}=\mathrm{CH}_{3}\end{array}$ & 96.3 & 2 & $\begin{array}{l}1.118-1.166(\mathrm{~m}, 3 \mathrm{H}) ; 2.093- \\
2.096(\mathrm{~d}, 3 \mathrm{H}) ; 2.276(\mathrm{~s}, 3 \mathrm{H}) \\
2.543(\mathrm{~s}, 3 \mathrm{H}) ; 3.616(\mathrm{~s}, 3 \mathrm{H}) \\
4.104-4.170(\mathrm{~m}, 2 \mathrm{H}) ; 6.008(\mathrm{~s}, \\
1 \mathrm{H})\end{array}$ & 53.04 & 6.12 & 19.03 \\
\hline $6 \mathrm{~d}$ & $\begin{array}{c}\mathrm{R}=\mathrm{CO}_{2} \mathrm{Et} ; \mathrm{R}^{1}=\mathrm{SMe} \\
\mathrm{R}^{3}, \mathrm{R}^{5}=\mathrm{CH}_{3} \\
\mathrm{R}^{4}=\mathrm{H} ; \mathrm{R}^{6}=\mathrm{COCH}_{3}\end{array}$ & 94.8 & 2 & $\begin{array}{l}1.115-1.174(\mathrm{~m}, 3 \mathrm{H}) ; 2.097- \\
2.134(\mathrm{~d}, 3 \mathrm{H}) ; 2.259-2.266(\mathrm{~d}, \\
3 \mathrm{H}) ; 2.554-2.571(\mathrm{~d}, 3 \mathrm{H}) ; 2.637 \\
(\mathrm{~s}, 3 \mathrm{H}) ; 4.136-4.190(\mathrm{~m}, 2 \mathrm{H}) \\
6.003(\mathrm{~s}, 1 \mathrm{H})\end{array}$ & 52.16 & 5.63 & 17.38 \\
\hline $6 e$ & $\begin{array}{c}\mathrm{R}=\mathrm{CN} ; \mathrm{R}^{1}=\mathrm{SCH}_{2} \mathrm{Ph} ; \\
\mathrm{R}^{3}, \mathrm{R}^{5}=\mathrm{CH}_{3} \\
\mathrm{R}^{4}=\mathrm{H} ; \mathrm{R}^{6}=\mathrm{CH}_{3}\end{array}$ & 87.9 & 2 & $\begin{array}{l}2.661-2.673(\mathrm{~d}, 3 \mathrm{H}) ; 2.800(\mathrm{~s} \\
3 \mathrm{H}) ; 3.940(\mathrm{~s}, 2 \mathrm{H}) ; 4.660-4.677 \\
(\mathrm{~d}, 3 \mathrm{H}) ; 6.807-6.830(\mathrm{~d}, 1 \mathrm{H}) \\
7.195-7.425(\mathrm{~m}, 5 \mathrm{H})\end{array}$ & 63.13 & 5.30 & 21.65 \\
\hline $6 f$ & $\begin{array}{c}\mathrm{R}=\mathrm{CO}_{2} \mathrm{Et} ; \mathrm{R}^{1}=\mathrm{SMe} \\
\quad \mathrm{R}^{3}, \mathrm{R}^{5}=\mathrm{H}, \mathrm{CF}_{3} \\
\mathrm{R}^{4}=\mathrm{H} ; \mathrm{R}^{6}=\mathrm{CH}_{3}\end{array}$ & 83.7 & 4 & $\begin{array}{l}1.370-1.416(\mathrm{t}, 3 \mathrm{H}) ; 2.514(\mathrm{~s}, \\
3 \mathrm{H}) ; 3.325-3.389(\mathrm{~d}, 1 \mathrm{H}) ; 3.573- \\
3.636(\mathrm{~d}, 1 \mathrm{H}) ; 3.794(\mathrm{~s}, 3 \mathrm{H}) ; \\
4.282-4.397(\mathrm{~m}, 2 \mathrm{H})\end{array}$ & $\begin{array}{l}43.15 \\
43.11\end{array}$ & 4.02 & 16.61 \\
\hline $6 \mathrm{~g}$ & $\begin{array}{c}\mathrm{R}=\mathrm{CO}_{2} \mathrm{Et} ; \mathrm{R}^{1}=\mathrm{SMe} \\
\mathrm{R}^{3}, \mathrm{R}^{5}=\mathrm{CH}_{3} \\
\mathrm{R}^{4}=\mathrm{H} ; \mathrm{R}^{6}=\mathrm{SO}_{2} \mathrm{CH}_{3}\end{array}$ & 86.3 & 2 & $\begin{array}{l}1.101-1.170(\mathrm{~m}, 3 \mathrm{H}) ; 2.134(\mathrm{~s}, \\
3 \mathrm{H}) ; 2.258(\mathrm{~s}, 3 \mathrm{H}) ; 2.587(\mathrm{~s}, \\
3 \mathrm{H}) ; 3.486(\mathrm{~s}, 3 \mathrm{H}) ; 4.143-4.182 \\
(\mathrm{q}, 2 \mathrm{H}) ; 6.006(\mathrm{~s}, 1 \mathrm{H})\end{array}$ & 43.56 & 5.04 & 15.53 \\
\hline $6 \mathrm{~h}$ & $\begin{array}{l}\mathrm{R}=\mathrm{CO}_{2} \mathrm{Et} ; \mathrm{R}^{1}=\mathrm{SMe} \\
\quad \mathrm{R}^{3}, \mathrm{R}^{5}=\mathrm{H}, \mathrm{NH}_{2} \\
\mathrm{R}^{4}=\mathrm{CO}_{2} \mathrm{Et} ; \mathrm{R}^{6}=\mathrm{CH}_{3}\end{array}$ & 98.7 & 4 & $\begin{array}{l}1.141-1.367(\mathrm{~m}, 6 \mathrm{H}) ; 2.525(\mathrm{~d}, \\
3 \mathrm{H}) ; 3.666(\mathrm{~d}, 3 \mathrm{H}) ; 3.486(\mathrm{~s}, \\
3 \mathrm{H}) ; 4.158-4.300(\mathrm{q}, 2 \mathrm{H}) ; 6.006 \\
(\mathrm{~s}, 2 \mathrm{H}) ; 7.843(\mathrm{~d}, 1 \mathrm{H})\end{array}$ & 47.54 & 5.57 & 19.76 \\
\hline
\end{tabular}


Table 3. Continued

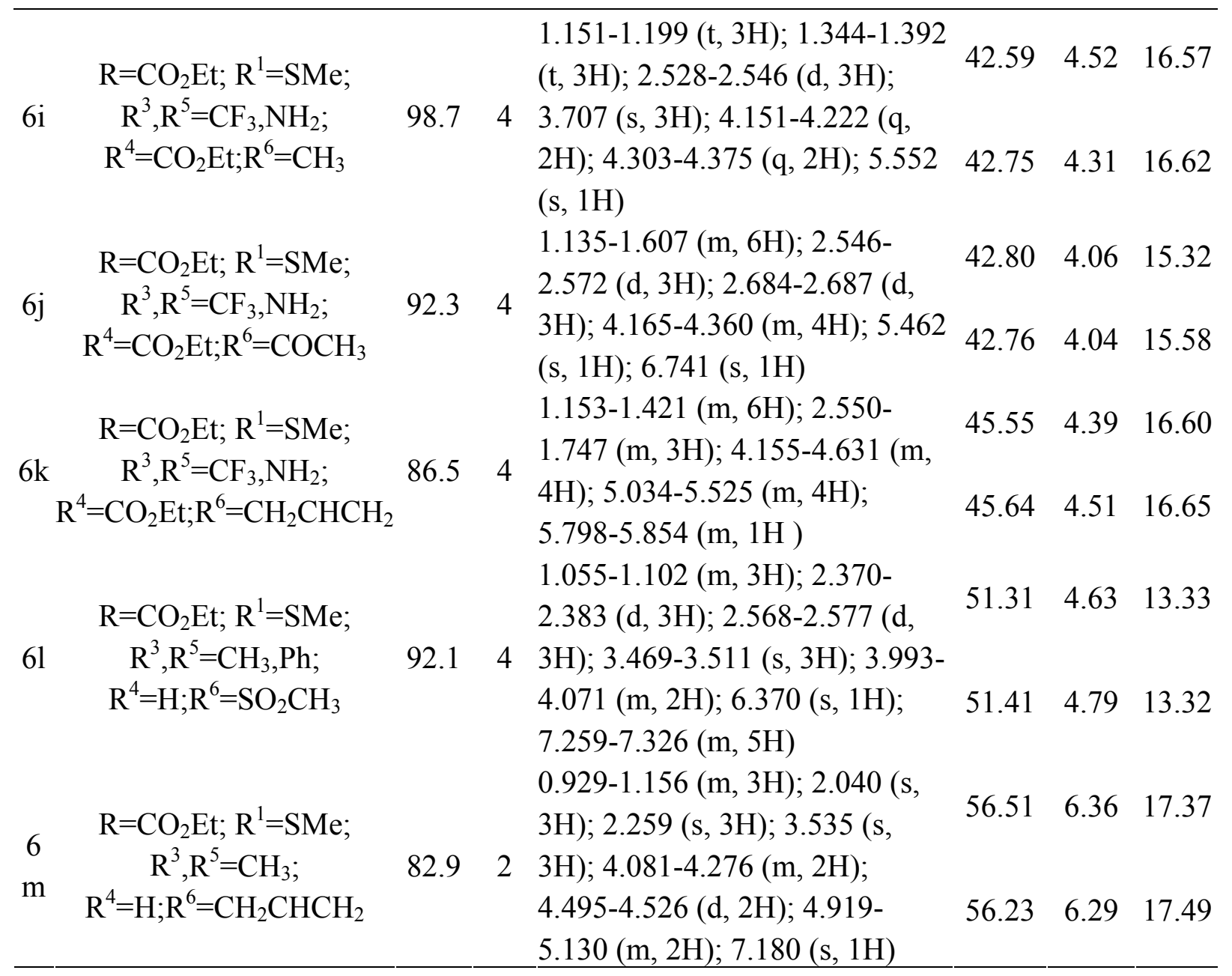

\section{Experimental Section}

General Procedures. Melting points were taken on a Yanaco-241 melting point apparatus and are uncorrected. The elemental analyses were done with Yanaco MT-3CHN apparatus. Proton magnetic resonance spectra were measured on a Bruker AC-P200 or Mercury-300BB instrument from solution in deuteriochloroform $\left(\mathrm{CDCl}_{3}\right)$ with tetramethylsilane (TMS) as the internal reference standard. Chemical shifts are reported as $\delta$-values in ppm downfield from TMS $\left(\delta_{\mathrm{TMS}}=\right.$ $0 \mathrm{ppm}$ ). Thin-layer chromatography (TLC) was carried out using precoated silica gel GF254 $(10 \sim 40 \mu)$ plates. Spots were visualized with a UV handlamp. Infrared (IR) spectra were recorded on an EQUINOX55 instrument. All samples were run as a thin film on potassium bromide plates. Absorption maxima were recorded in wave numbers $\left(\mathrm{cm}^{-1}\right)$. Electron impact (E.I.) mass spectra were recorded on a VG ZAB-HS instrument. 
5-Amino-1H-pyrazole (2). Compound 1 reacted with equimolecular hydrazine hydrate in ethanol and tetrahydrofuran at room temperature until the raw material disappeared from the TLC. The solvent was removed under reduced pressure and the product, 2 obtained was recrystallized from propan-2-ol.

Pyrazole hydrazine salt (3). 5-Amino- $1 H$-pyrazole $2(0.58 \mathrm{mmol})$ was dissolved in concentrated hydrochloric acid, and sodium nitrite $(0.66 \mathrm{mmol})$ in water was added dropwise at $0{ }^{\circ} \mathrm{C}$. The mixture was stirred for $2 \mathrm{~h}$ at $0{ }^{\circ} \mathrm{C}$ to achieve the corresponding diazonium salt of 2 .

The pyrazolyldiazonium salt was added to a solution of saturated aqueous $\mathrm{Na}_{2} \mathrm{SO}_{3}$. After stirring at room temperature for $2 \mathrm{~h}$, the reaction mixture was heated to $70{ }^{\circ} \mathrm{C}$, and then acidified using concentrated hydrochloric acid and adjusted at $\mathrm{pH}$ 2. The reaction mixture was stirred at this temperature for additional $4 \mathrm{~h}$. The resulting precipitate 3 was filtered off and used in the next reaction without further purification.

Substituted pyrazolo[1,5-a]pyrimidines (4). A solution of 2 in acetic acid was refluxed with equimolar bifunctional compound for $5 \mathrm{~h}$. After completion of the reaction, the solution was poured into ice-water. The white solid formed was filtered, and purified by crystallization from acetone.

1-H Pyrazolylpyrazoles (5). A solution of compound 3 (1.0 mmol), bifunctional compound $(1 \mathrm{mmol})$ and potassium carbonate $(1.0 \mathrm{mmol})$ in ethanol was refluxed for $5 \mathrm{~h}$. The reaction mixture was evaporated to dryness under reduced pressure. Trituration of residue with ethanol gave 5 .

1-Substituted pyrazolylpyrazoles (6). To a solution of compounds 5 (0.36 mmol) and potassium carbonate $(0.36 \mathrm{mmol})$ in $7 \mathrm{ml}$ of acetone, substituted regent, such as $\mathrm{CH}_{3} \mathrm{I}$ $(0.43 \mathrm{mmol})$ in $3 \mathrm{ml}$ of acetone was added dropwise at room temperature and the mixture was stirred over night at the same temperature. When the reaction was completed, the solvent was removed in vacuo and the residue was washed with water. Crude product was obtained without further purification in high yield.

\section{References and Footnotes}

1. Hartfiel, U.; Dorfmeister, G.; Franke, H.; Geisler, J.; Johann, G.; Rees, R. U.S. Patent 5,405,829, 1995.

2. Fraley, M. E.; Hoffman, W. F.; Rubino, R. S.; Hungate, R. W.; Tebben, A. J.; Rutledge, R. Z.; McFall, R. C.; Huckle, W. R.; Kendall, R. L.; Coll, K. E.; Thomas, K. A. Bioorganic \& Medicinal Chemistry Letters 2002, 12, 2767.

3. Zou X. M. Ph.D. Thesis, Nankai University, 1999 (in Chinese).

4. Wastrow, D. J.; Capiris, T.; Rubin, R.; Knobelsdorf, J. A.; Akunne, H.; Davis, M. D.; MacKenzie, R.; Pugsley, T. A.; Zoski, K. T.; Heffner, T. G.; Wise, L. D. Bioorganic \& Medicinal Chemistry Letters 1998, 8, 2067. 
5. Sawhney, S. N.; Tomer, R. K.; Prakash, O. M.; Prakash, I.; Singh, S. P. Indian Journal of Chemistry 1981, 20B, 314.

6. Aiello, E.; Aiello, S.; Mingoia, F.; Bacchi, A.; Pelizzi, G.; Musiu, C.; Setzu, M. G.; Pani, A.; Colla, P. L.; Marongiu, M. E. Bioorganic \& Medicinal Chemistry 2000, 8, 2719.

7. Marcel S. F.; Jie, L. K.; Lau, M. L. Chemistry and Physics of Lipids 1999, 101, 237.

8. Sloop, J. C.; Bumgardner, C. L.; Loehle, W. D. Journal of Fluorine Chemistry 2002, 118, 135.

9. Plate, R.; Plaum, M. J. M.; Boer, T. D.; Andrews, J. S.; Rae, D. R.; Gibson, S. Bioorganic \& Medicinal Chemistry 1996, 4, 227.

10. Ren X. L.; Wu C.; Hu F. Z.; Zou X. M.; Yang H. Z. Chinese Journal of Chemistry 2004, 22, 194. 